\title{
Neamine is preferential as an anti-prostate cancer reagent by inhibiting cell proliferation and angiogenesis, with lower toxicity than cis-platinum
}

\author{
YA-PING LIU ${ }^{1}$, GUO-FU HU ${ }^{2}$ and YUN-XIA WU ${ }^{1}$ \\ ${ }^{1}$ School of Pharmacy, Tongji Medical College, Huazhong University of Science and Technology, Wuhan, Hubei 430030, \\ P.R. China; ${ }^{2}$ Molecular Oncology Research Institute, Tufts Medical Center, Boston, MA 02111, USA
}

Received September 20, 2014; Accepted April 30, 2015

DOI: $10.3892 / \mathrm{ol} .2015 .3227$

\begin{abstract}
Hormone therapy is the most commonly used treatment for prostate cancer, but for androgen-independent cancer, few effective treatment methods are available. Therefore, the requirement to develop novel and effective anti-prostate cancer drugs is extremely urgent. Angiogenin has been suggested as a molecular target for prostate cancer treatment; its overexpression contributes to androgen-dependent prostate cancer growth and castration-resistant growth of androgen-independent prostate cancer. The aim of the present study was to investigate whether neamine, a low toxicity angiogenin nuclear translocation inhibitor, has preferential anti-prostate cancer activity compared with cis-platinum (DDP) and the mechanisms involved. Immunofluorescence and MTT assays were used to observe the effect of neamine on the nuclear translocation of angiogenin and cell proliferation, and a PC-3 cell transplanted tumor model was used to investigate the in vivo activity of neamine and DDP. Immunohistochemistry was performed to observe the expression of angiogenin, cluster of differentiation (CD)31 and Ki-67. It was found that neamine blocked nuclear translocation of angiogenin effectively and inhibited angiogenin-induced human umbilical vein endothelial cell and PC-3 cell proliferation in a dose-dependent manner. Neamine exerted a comparative antitumor effect, but lower toxicity (weight loss), in the PC-3 xenograft models treated with DDP. Neamine consistently reduced the expression of angiogenin and CD31 significantly, but no difference was found in Ki-67 expression compared with DDP. These data suggested that neamine may be a promising agent for prostate cancer treatment.
\end{abstract}

Correspondence to: Professor Yun-Xia Wu, School of Pharmacy, Tongji Medical College, Huazhong University of Science and Technology, 13 Aviation Road, Wuhan, Hubei 430030, P.R. China E-mail:wuyunxia@hust.edu.cn

Key words: neamine, cis-platinum, angiogenin, PC-3 xenograft tumor model

\section{Introduction}

Prostate cancer is the most prevalent malignancy and the second leading cause of cancer-related mortality in men worldwide (1). A total of 233,000 new cases and 29,480 associated mortalities are projected to occur in the United States in 2014 (2). Current therapy strategies for prostate cancer mainly include radical prostatectomy, external beam radiation, cryotherapy, chemotherapy or hormonal therapy. Among these, hormonal therapy is the most commonly used method, while for androgen-independent cancer, few effective treatment methods are available (3). Therefore, the requirement to develop novel and effective anti-prostate cancer drugs is extremely urgent.

Angiogenesis is an essential step for the formation, progression and metastasis of numerous types of cancer, including prostate cancer (4). Previous studies have reported that the level of several angiogenic factors, such as vascular endothelial growth factor (VEGF) (5), transforming growth factor- $\beta$ (6), fibroblast growth factors (FGFs) (7) and cyclooxygenase-2 (8), are upregulated in prostate cancer. An increasing number of studies are now focusing on angiogenin, a 14.4-kDa angiogenic ribonuclease, which was firstly isolated from the conditioned medium of human colon adenocarcinoma HT-29 cells based on its angiogenic activity, and which plays a controlling role in tumor angiogenesis (9). Angiogenin can transfer to the nucleus to stimulate rRNA transcription, which is an indispensable process in angiogenesis and cell proliferation pathways, and is also a crossroad for angiogenesis induced by acidic and basic FGFs ( $\alpha$ FGF, $\beta$ FGF), VEGF and epidermal growth factor $(10,11)$. In addition, angiogenin was reported to have an anti-apoptotic effect by targeting p53 and B-cell lymphoma 2, thereby accelerating cancer development (12). Angiogenin is upregulated in a number of cancers, including prostate cancer. It has been demonstrated that the plasma angiogenin level is elevated in prostate cancer patients, particularly in hormonal refractory prostate cancer patients (13), and that serum angiogenin may be used as a prostate cancer diagnostic tool among candidates for biopsy (14). Katona et al (15) reported that angiogenin expression increased as prostate cancer progressed from a benign phenotype to invasive adenocarcinoma. Using immunohistochemistry, Yoshioka et al (16) found that strong 
staining for angiogenin was present in the extracellular matrix (ECM), cytoplasm and nucleus of prostate cancer tissues, but that no staining was present in the cytoplasm and nucleus of normal prostate tissues, with strong staining in the ECM. Earlier studies indicated that angiogenin antagonists could prevent HT-29 tumor appearance and growth $(17,18)$. It is conceivable that an inhibitor targeted to angiogenin may achieve ideal effects for the treatment of prostate cancer.

Neomycin, an aminoglycoside antibiotic, was firstly found to have an inhibitory effect on angiogenin nuclear translocation in human umbilical vein endothelial cells (HUVECs) (19), but its use as a chemotherapeutic agent is limited due to its nephrotoxicity and ototoxicity. Notably, as a derivative of neomycin, neamine has equivalent effects but less toxicity than neomycin. Previous studies demonstrated that neamine could inhibit the proliferation of human hepatoma H7402 cells (20) and human oral cancer HSC-2 cells (21). Furthermore, it suppressed the growth of the established A549 (22) HT-29, MDA-MB-435, A431 (23), HSC-2 and SAS (21) tumor transplants, and the formation of Kaposi's sarcoma-associated herpesvirus-positive primary effusion lymphoma (12).

The human prostate cell line PC-3, is a hormone-independent cell line. Hormone therapy is the most commonly used treatment for prostate cancer; however, chemotherapy is available for androgen-independent cancer. Cis-platinum (DDP) is an effective chemotherapeutic drug for prostate cancer, and there are currently no small-molecule targeted inhibitors for prostate cancer, so the present study selected DDP as a positive comparison. The present study investigated the anti-prostate cancer activity of neamine compared with DDP, and the mechanism behind this.

\section{Materials and methods}

Preparation of neamine. Neamine was obtained from neomycin through alcoholysis (24). Briefly, $90 \mathrm{~g}$ neomycin sulfate (Sanxia Pharmaceutical Factory, Yichang, Hubei, China) was reflux in 3 liters of methanol with $1 \mathrm{mM} \mathrm{HCl}$ for $10 \mathrm{~h}$. Next, $40 \mathrm{ml}$ ammonia and 3 liters of methanol were added to conduct ammonification for $2 \mathrm{~h}$. The precipitate was washed with methanol, purified by recrystallization in an ethanol:water mixture (9:1) and then dried in an oven; $13 \mathrm{~g}$ neamine was obtained. The structure of neamine (Fig. 1) was confirmed by nuclear magnetic resonance spectroscopy (Bruker Corporation, Beijing, China) and the purity (99.67\%) was determined by evaporative light scattering detector-high performance liquid chromatography (Waters Corporation, Milford, MA, USA).

Cell lines, reagents and mice. HUVECs and the human prostate cancer PC-3 cell line were kindly provided by the Gynecological Oncology Center of Tongji Hospital (Wuhan, China). The HUVECs were cultured in ECM medium (ScienCell, Carlsbad, CA, USA) with $5 \%$ fetal bovine serum (FBS), $1 \%$ endothelial cell growth supplement and $1 \%$ penicillin-streptomycin, and the PC-3 cells were cultured in RPMI 1640 medium (GE Healthcare, Logan, UT, USA) supplemented with $10 \%$ FBS. Angiogenin and rabbit anti-human angiogenin polyclonal antibody (R113, $10 \mu \mathrm{g} . \mathrm{ml})$ were donated by Professor Guo-Fu Hu (Molecular Oncology Research Institute, Tufts Medical

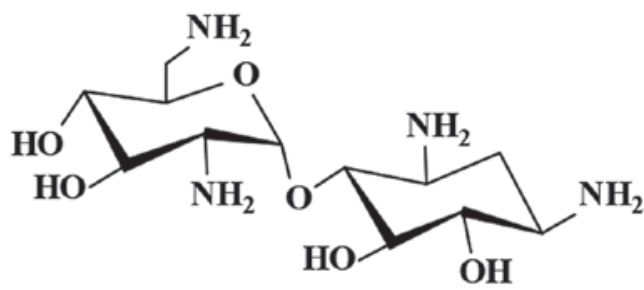

\section{Neamine}

Figure 1. Structure of neamine.

Center, Boston, MA, USA). Cluster of differentiation (CD)31 rabbit polyclonal antibody (cat no. ab28364; 1:2,000) and Ki-67 mouse monoclonal antibody (clone, MIB-1; 1:2,000) were purchased from Abcam (Cambridge, UK) and Dako (Glostrup, Denmark), respectively. DDP was produced by Qilu Pharmaceutical Factory (Shandong, China). The 4-week-old, specific pathogen-free (SPF), male Balb/c nude mice (certificate number, 43004700004854) were provided by the Human SJA Laboratory Animal Co. Ltd., (Changsha, China) and maintained in the SPF-level Experimental Animal Center of Huazhong University of Science and Technology (Wuhan, China).

Nuclear translocation of angiogenin. The HUVEC cells or PC-3 cells $\left(1 \times 10^{4}\right.$ per well) were cultured in 24 -well plates with slides for $24 \mathrm{~h}$ and treated with $100 \mu \mathrm{mol}$ neamine in the presence or absence of $1 \mu \mathrm{g} / \mathrm{ml}$ angiogenin for $40 \mathrm{~min}$ at $37^{\circ} \mathrm{C}$. The cells were then fixed with methanol at $-20^{\circ} \mathrm{C}$ for $15 \mathrm{~min}$. The fixed cells were blocked with 5\% bovine serum albumin in phosphate-buffered saline (PBS) and then incubated with $10 \mu \mathrm{g} / \mathrm{ml}$ rabbit anti-human angiogenin polyclonal antibody R113 at $4^{\circ} \mathrm{C}$ overnight, washed three times with PBS and incubated with fluorescein isothiocyanate-labeled goat anti-rabbit immunoglobulin $\mathrm{G}(1: 100)$ at room temperature for $1 \mathrm{~h}$ in the dark. The cells were washed, dyed with Hoechst 33342, mounted with 50\% glycerol and observed under a confocal laser scanning microscope (Nikon, Tokyo, Japan).

Cell viability assay. The HUVEC or PC-3 cells were seeded into 96 -well plates $(5,000$ cells/well) and treated with $1 \mu \mathrm{g} / \mathrm{ml}$ angiogenin in the presence of various concentrations of neamine $(0,10,25,50100$ and $200 \mu \mathrm{mol})$ for $48 \mathrm{~h}$. After $4 \mathrm{~h}$ of incubation with MTT, $150 \mu \mathrm{l}$ DMSO was added to dissolve the crystals. The absorbance was determined by a Synergy HT plate reader (Biotek Instruments Inc., Winooski, VT, USA) at $490 \mathrm{~nm}$. The cell viability was calculated relative to the control cells. The assay was repeated three times.

In vivo study on the growth of $P C-3$ cell tumor xenografts. This study was conducted at the SPF-level Experimental Animal Center of Huazhong University of Science and Technology. All animal protocols were in accordance with the Chinese animal protection laws and guidelines for the use of living animals for scientific purposes and were approved by the Ethical Committees of Tongji Medical College, Huazhong University of Science and Technology. All mice were allowed to acclimate to new surroundings for 5 days prior to the experiments. 


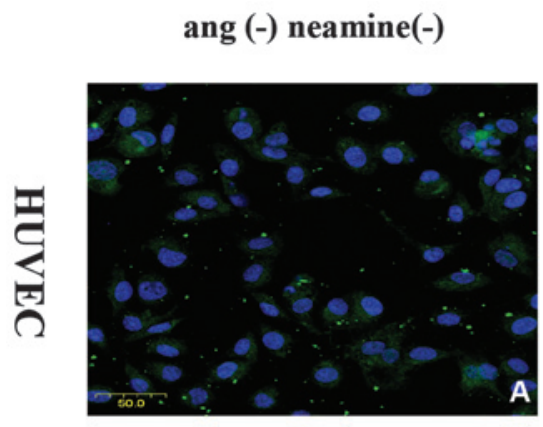

ang (+) neamine(-)

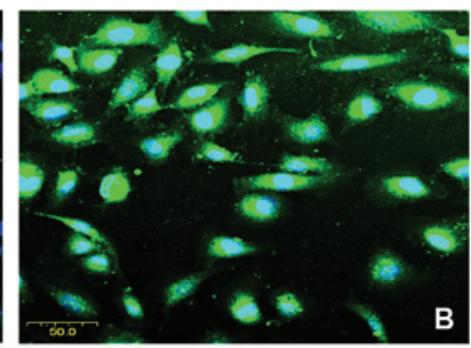

B
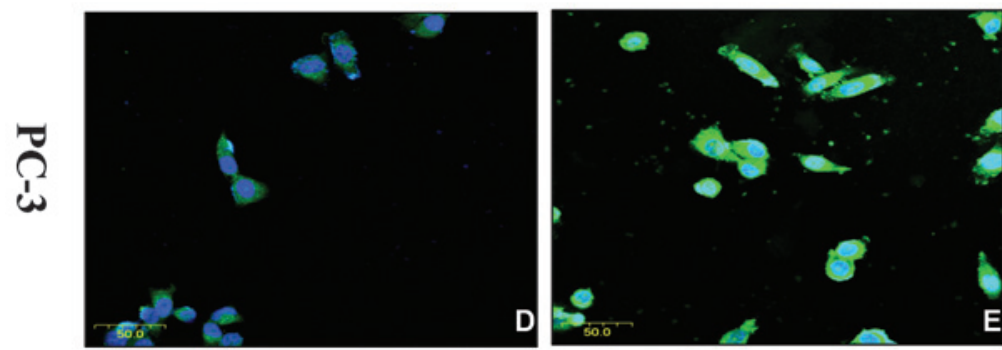

ang $(+)$ neamine $(+)$
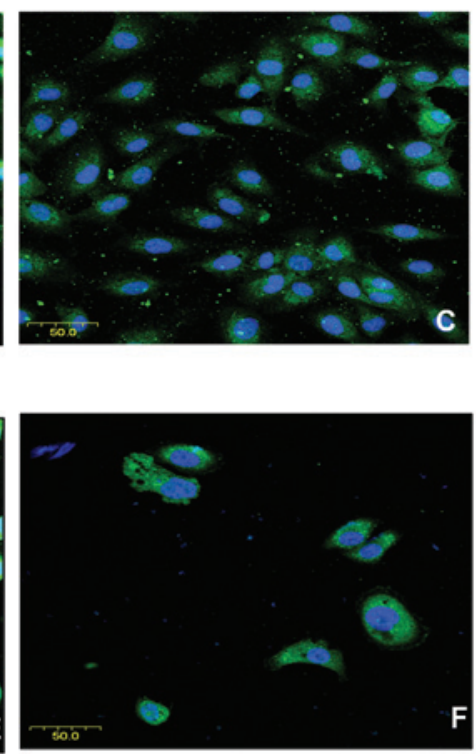

Figure 2. Neamine blocks the translocation of angiogenin in human umbilical vein endothelial cells (HUVECs) and PC-3 cells. HUVECs and PC-3 cells were respectively incubated in extracellular matrix (ECM) supplement medium and RPMI 1640 medium for $24 \mathrm{~h}$. The cells were then washed with phosphate-buffered saline, treated with (A) ECM medium or (D) RPMI 1640 medium; (B and E) $1 \mu \mathrm{g} / \mathrm{ml}$ angiogenin; or (C and F) $1 \mu \mathrm{g} / \mathrm{ml}$ angiogenin and $100 \mu$ mol neamine at $37^{\circ} \mathrm{C}$ for $40 \mathrm{~min}$. The fixed cells were incubated in the appropriate primary and secondary antibody. Magnification, $\mathrm{x} 400$.

A total of $5 \times 10^{6} \mathrm{PC}-3$ cells resuspended in $200 \mu 1$ serum-free RPMI 1640 medium and 50\% Matrigel (BD Biosciences, Franklin Lakes, NJ, USA) were injected subcutaneously into the male Balb/c nude mice. When the average tumor volume reached $\sim 100 \mathrm{~mm}^{3}$, the mice were randomly divided into three groups ( $n=6 /$ group) and treated with an injection of saline into the tail vein, DDP (2 mg/kg, every other day, 6 times) and neamine (15 mg/kg, every day, 12 times). The body weights of the mice, and the length and width of the tumors were measured every 3 days. The tumor volume (V) was calculated according to the formula: $\mathrm{V}=$ length $\mathrm{x}$ width $^{2} / 2$, while the body weight change rate was expressed by $\mathrm{W}_{\mathrm{n}} / \mathrm{W}_{1} \times 100$, in which Wn and W1 represented the body weights measured at the corresponding day and the first day, respectively.. After 12 days of continuous administration, the mice were sacrificed sacrificed by cervical vertebra dislocation, and the tumors were isolated and weighted.

Immunohistochemistry. Immunohistochemistry was performed as previously described (25), with the following modifications, sections were incubated with $10 \mu \mathrm{g} / \mathrm{ml}$ R113 antibody, CD31 (1:2,000) polyclonal antibody and Ki-67 monoclonal antibody (1:200). Angiogenin was stained with R113 antibody, neovessels were stained with CD31 and proliferating cells were stained with Ki-67 monoclonal antibody. The mean positive staining density was analyzed in 5 randomly selected areas in each section at x400 magnification using image analysis software (Image-Pro plus 7.0; Media Cybernetics, Inc., Rockville, MD, USA).

Statistical analysis. All tests were performed using the SPSS 20.0 statistical software package (IBM SPSS, Armonk, NY, USA). Data are presented as the mean \pm standard deviation. Analysis of variance and Student's t-test were used to evaluate statistical significance, and $\mathrm{P}<0.05$ was considered to indicate a statistically significant difference.

\section{Results}

Neamine blocks the translocation of angiogenin. Immunofluorescence was used to observe the effects of neamine on the translocation of angiogenin. As shown in Fig. 2, it presented stronger staining of angiogenin in the nucleus of the HUVECs (Fig. 2B) and PC-3 cells (Fig. 2E) when exogenous angiogenin was added compared with the control group without exogenous angiogenin stimulation (Fig. 2A and D). In the presence of $100 \mu \mathrm{mol}$ neamine (Fig. 2C and F), the nuclear expression of angiogenin was markedly decreased.

Neamine reduces cell viability in HUVEC and PC-3 cells. As shown in Fig. 3, it was demonstrated that exogenous angiogenin promoted HUVEC and PC-3 cell proliferation, and that neamine inhibited angiogenin-mediated HUVEC and PC-3 cell proliferation and viability in a dose-dependent manner in the range of 10-200 $\mu \mathrm{mol}$. With regard to the HUVECs, exogenous angiogenin promoted $37.4 \%$ cell proliferation compared with the control wells (without angiogenin), and $50 \mu$ mol neamine completely inhibited angiogenin-induced cell proliferation. However, for the PC-3 cells, in the presence of angiogenin, there was only a $15 \%$ cell proliferation promotion rate, which was lower than that in the HUVECs. Additionally, $50 \mu \mathrm{mol}$ neamine inhibited angiogenin-induced proliferation activity by $75 \%$, and the full inhibition was achieved at $200 \mu \mathrm{mol}$ neamine.

Neamine has a comparative antitumor effect, but lower toxicity (weight loss), compared with DDP in PC-3 xenograft models. As shown in Fig. 4A, continuous intravenous 

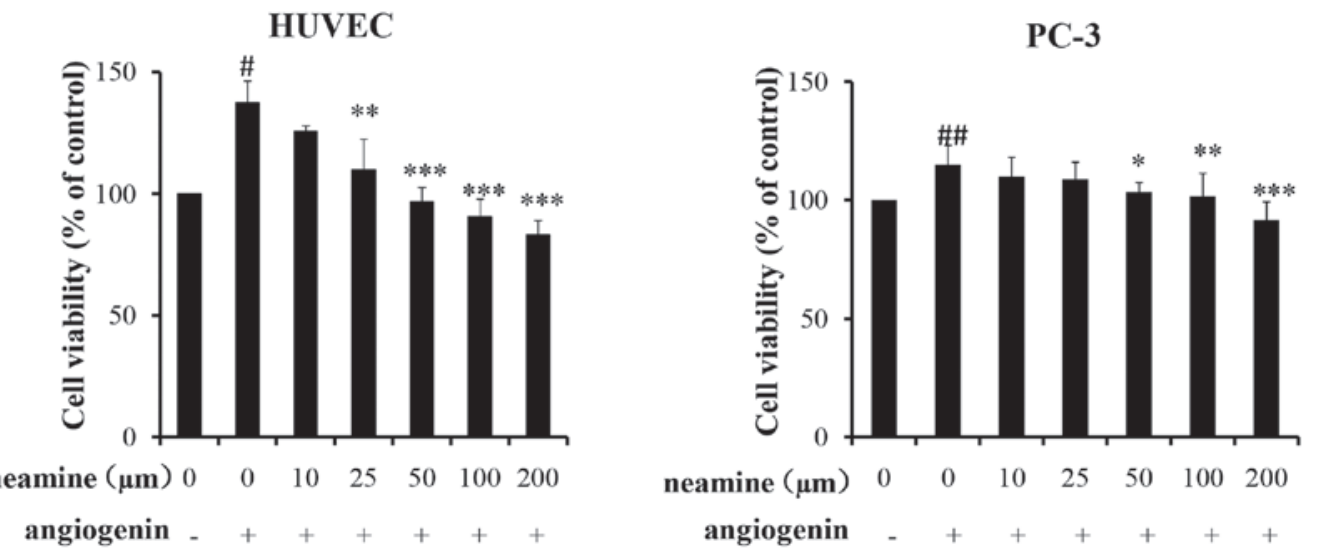

Figure 3. Inhibition of human umbilical vein endothelial cell (HUVEC) and PC-3 proliferation by neamine. HUVEC and PC-3 cells were treated with a gradient concentration of neamine in the presence or absence of $1 \mu \mathrm{g} / \mathrm{ml}$ angiogenin for $48 \mathrm{~h}$. The percentage of cell viability at given concentrations compared with untreated control cells was measured according to MTT assay. Data are presented as the mean \pm standard deviation. ${ }^{*} \mathrm{P}<0.05,{ }^{* *} \mathrm{P}<0.01$ and ${ }^{* * * *} \mathrm{P}<0.001$ vs. angiogenin wells; ${ }^{\#} \mathrm{P}<0.05$ and ${ }^{\# \#} \mathrm{P}<0.01$ vs. control wells.
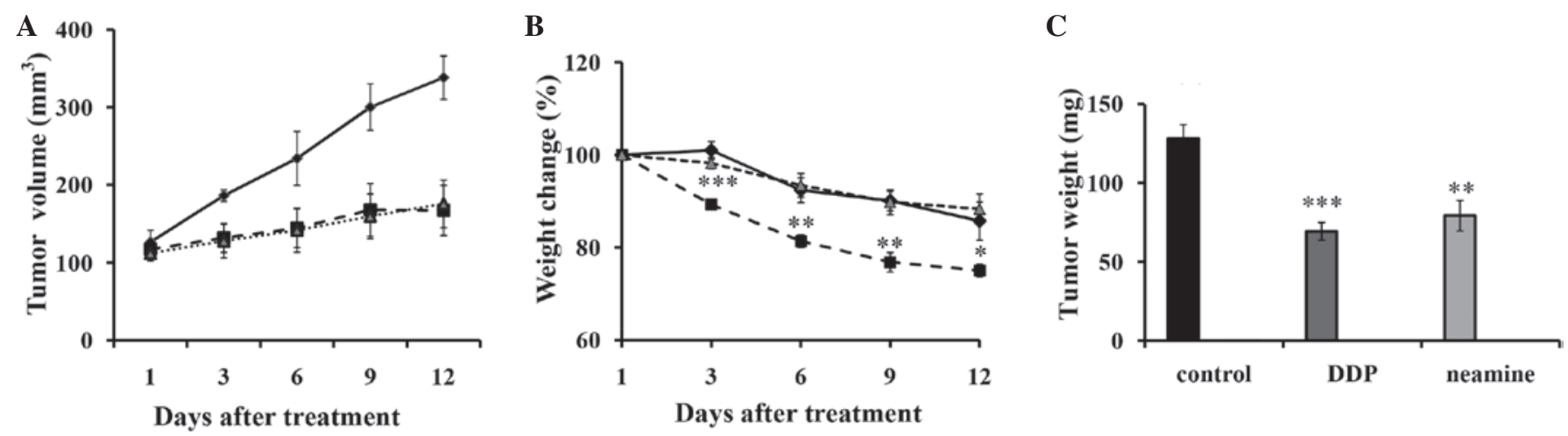

Figure 4. Neamine displays in vivo inhibition of tumor growth in PC-3 cell xenograft models. PC-3 cells were inoculated into the left flank of nude mice, and treatment was started on day 5 post-inoculation. (A) Tumor volume of the saline control group (- $\bullet$-), the cis-platinum (DDP) group (-----) and the neamine group (----) at different post-treatment times. (B) Changes in body weight of the tumor-bearing mice in each group. Tumor sizes and body weight were measured every 3 days. (C) Tumor weight of each group at the end of the experiment. Values are presented as the mean \pm standard deviation. ${ }^{*} \mathrm{P}<0.05$, ${ }^{* *} \mathrm{P}<0.01$ and ${ }^{* * *} \mathrm{P}<0.001$ vs. saline control group.

administration of DDP and neamine significantly suppressed the tumor size and volume from 3 days post-treatment until the end of the experiment. As indicated in Fig. 4B, an irregular decrease in body weight was presented in all groups, and there was a significant difference between DDP and the saline control group during the whole process, while no marked difference was observed between the neamine group and the saline control group. The average weight of the harvested tumors in the saline group was $128 \pm 8.86 \mathrm{mg}$ (Fig. 4C), whereas the average tumor weights in the DDP and neamine groups were $69.38 \pm 5.51$ and $79.26 \pm 9.59 \mathrm{mg}$, respectively. There was no marked difference in tumor growth and weight between the neamine and DDP groups, but lower toxicity (weight loss) was found in the neamine group compared with the DDP.

Expression of angiogenin, CD31 and Ki-67 in tumors in the DDP and neamine groups. As shown in Fig. 5, strong angiogenin staining was present in the nucleus and cytoplasm of the tumors from the saline control group; the expression was focused mainly in the cytoplasm, with little in the nucleus following neamine-treatment, but strong nuclear expression was still present in the tumors of the DDP group, showing that neamine effectively restrained the nuclear translocation of angiogenin. The mean density of CD31 in the saline control, DDP and neamine groups was $0.41 \pm 0.06,0.37 \pm 0.09(\mathrm{P}=0.512$ vs. control $)$ and $0.11 \pm 0.01$ ( $\mathrm{P}=0.002$ vs. control; $\mathrm{P}=0.003$ vs. $\mathrm{DDP})$, respectively, indicating that neamine decreased tumor angiogenesis, but that DDP exhibited no significant effect on tumor angiogenesis. The percentage of Ki-67-positive cells was $55.23 \pm 3.36 \%$ in the saline control group, $12.97 \pm 2.21 \%(\mathrm{P}<0.001$ vs. control, $\mathrm{P}=0.009$ vs. neamine) in the DDP group and $21.43 \pm 2.59 \%$ $(\mathrm{P}<0.001$ vs. control) in the neamine group, which represented a 76.5 and $61.2 \%$ decrease in tumor cell proliferation in the DDP and neamine groups, respectively.

\section{Discussion}

Angiogenin, as a key angiogenic factor, can transfer into the cell nucleus and then bind to the rRNA gene to stimulate rRNA transcription (11), which is essential for angiogenesis and cell proliferation. The elevated expression of angiogenin has been reported in a number of cancer types, and prostate cancer is 

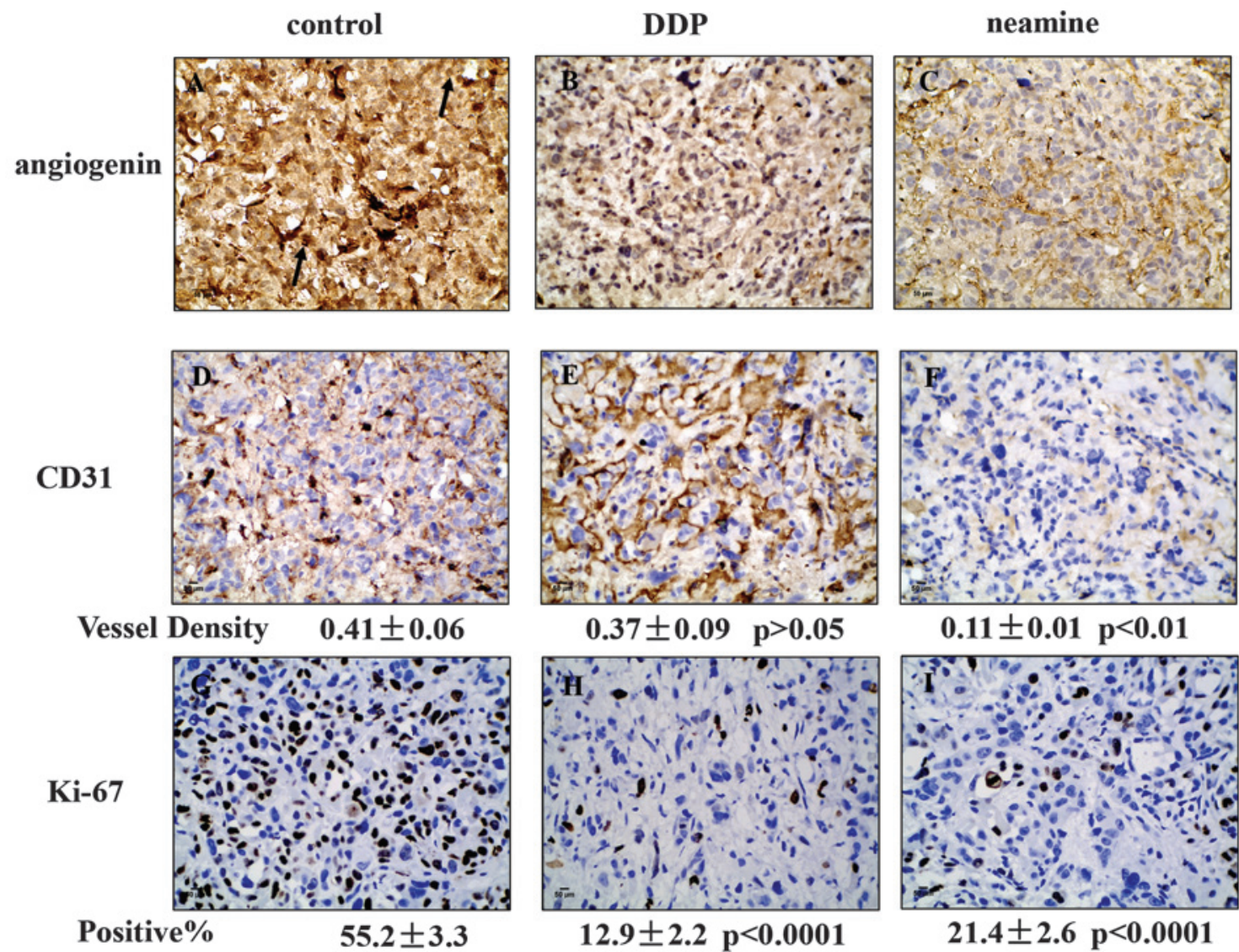

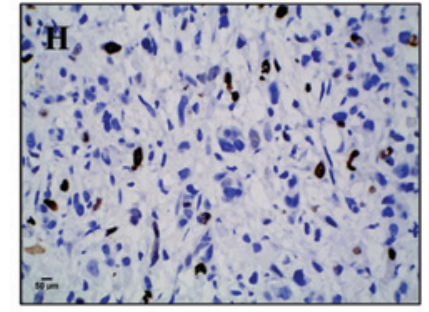

$12.9 \pm 2.2 p<0.0001$

\section{$0.11 \pm 0.01 p<0.01$}

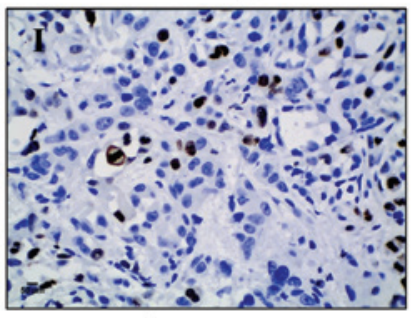

$21.4 \pm 2.6 p<0.0001$

Figure 5. Expression of angiogenin, cluster of differentiation (CD)31 and Ki-67 in the tumors. Sections (4 $\mu$ m) were cut and stained with (A-C) anti-human angiogenin antibody, (D-F) anti-CD31 antibody and (G-I) anti-Ki-67 antibody. The black arrows in (A) indicate the nuclear expression of angiogenin. Microvessel density and the Ki-67-positive rate were calculated in five areas at $\mathrm{x} 400$ magnification. The values are presented as the mean \pm standard deviation.

no exception. Previous studies indicated that the expression of angiogenin increased along with the progression of prostate cancer (15), and that the plasma angiogenin level was elevated in prostate cancer patients, particularly in hormonal refractory prostate cancer patients (13). Neomycin and neamine were found to inhibit the nuclear translocation of angiogenin (26), but neamine had much less toxicity than neomycin $(27,28)$. PC-3 cells are a type of hormone-independent prostate cancer cell, and in the present study, it was demonstrated that neamine blocked the translocation of angiogenin in the PC-3 cells, with an effect that was comparable to DDP in PC-3 xenografts, but with much lower toxicity. Therefore, neamine may hold great potential as a potent agent against prostate cancer, particularly the hormone-independent type.

In theory, the translocation of angiogenin could enhance rRNA transcription and further promote cell proliferation. The cell viability results in the present study indicated that angiogenin-stimulated cell proliferation of the HUVECs and PC-3 cells rather than basal level cell proliferation was inhibited by neamine, and also that the HUVECs were more sensitive to neamine than the prostate cancer PC-3 cell line. Thus, it may be concluded that neamine may not induce drug tolerance and cytotoxicity based on its main pertinence for endothelial cells and angiogenin upregulated cancer cells.

In the present study, it was confirmed that exogenous angiogenin underwent nuclear translocation in the PC-3 cells and HUVECs, and that neamine effectively blocked this process. The nuclear expression of angiogenin was significantly decreased in the neamine-treated tumors compared with the saline controls. Treatment with neamine decreased CD31 expression to a greater extent than DDP, but Ki-67 expression to a lesser extent than DDP. This proved that neamine exhibited a dual effect by suppressing tumor angiogenesis and cancer cell proliferation, but that DDP exhibited no marked effect on angiogenesis.

As observed in the present animal experiments, treatment with neamine or DDP inhibited the progression of established PC-3 transplanted tumors in Balb/c nude mice. However, although DDP achieved better results, it exhibited a bigger adverse impact on body weight and more side-effects compared with neamine in the whole process.

Angiogenesis is a major step for the growth, spread and metastasis of solid tumors, therefore anti-angiogenesis agents may have great potential in targeting malignancy. A variety of anti-angiogenesis drugs have been used in clinical or pre-clinical research, with the greatest success being Avastin, which has been approved to be used in the treatment of metastatic colorectal cancer (29). The present results showing the significant effect of neamine against prostate cancer also highlight the potential of neamine as an anti-angiogenesis drug. However, the combination of an anti-angiogenesis drug and a chemotherapeutic drug may be more effective in halting the progression of cancer. Just as indicated in Fig. 5, neamine exhibited a better effect on angiogenesis, but a weaker effect on cell proliferation compared with DDP, while DDP exhibited 
a better effect on cell proliferation, but a weaker effect on angiogenesis compared with neamine. Thus, future clinical experiments will investigate the synergistic effect of neamine and DDP against prostate cancer.

\section{Acknowledgements}

This study was supported by the National Major Special Project Foundation of China Ministry of Science and Technology (no. 2012ZX09103101047), the National Natural Science Foundation of China (no. 81373873) and the Central College Basic Scientific Research Business Special Fund (no. 2014QN129).

\section{References}

1. Greenlee RT, Hill-Harmon MB, Murray T and Thun M: Cancer statistics, 2001. CA Cancer J Clin 51: 15-36, 2001.

2. Siegel R, Ma J, Zou Z and Jemal A: Cancer statistics, 2014. CA Cancer J Clin 64: 9-29, 2014.

3. Santos AF, Huang $\mathrm{H}$ and Tindall DJ: The androgen receptor: A potential target for therapy of prostate cancer. Steroids 69: 79-85, 2004.

4. Izawa JI and Dinney CP: The role of angiogenesis in prostate and other urologic cancers: A review. CMAJ 164: 662-670, 2001.

5. Ferrer FA, Miller LJ, Andrawis RI, Kurtzman SH, Albertsen PC, Laudone VP and Kreutzer DL: Vascular endothelial growth factor (VEGF) expression in human prostate cancer: In situ and in vitro expression of VEGF by human prostate cancer cells. J Urol 157: 2329-2333, 1997.

6. Wikström P, Bergh A and Damber JE: Transforming growth factor-betal and prostate cancer. Scand J Urol Nephrol 34: 85-94, 2000.

7. Acevedo VD, Gangula RD, Freeman KW, et al: Inducible FGFR-1 activation leads to irreversible prostate adenocarcinoma and an epithelial-to-mesenchymal transition. Cancer Cell 12: 559-571, 2007.

8. Uotila P, Valve E, Martikainen P, Nevalainen M, Nurmi M and Härkönen P: Increased expression of cyclooxygenase-2 and nitric oxide synthase-2 in human prostate cancer. Urol Res 29: 23-28, 2001.

9. Fett JW, Strydom DJ, Lobb RR, et al: Isolation and characterization of angiogenin, an angiogenic protein from human carcinoma cells. Biochemistry 24: 5480-5486, 1985.

10. Moroianu J and Riordan JF: Nuclear translocation of angiogenin in proliferating endothelial cells is essential to its angiogenic activity. Proc Natl Acad Sci USA 91: 1677-1681, 1994.

11. Kishimoto K, Liu S, Tsuji T, Olson KA and Hu GF: Endogenous angiogenin in endothelial cells is a general requirement for cell proliferation and angiogenesis. Oncogene 24: 445-456, 2005.

12. Bottero V, Sadagopan S, Johnson KE, Dutta S, Veettil MV and Chandran B: Kaposi's sarcoma-associated herpesvirus-positive primary effusion lymphoma tumor formation in NOD/SCID mice is inhibited by neomycin and neamine blocking angiogenin's nuclear translocation. J Virol 87: 11806-11820, 2013.
13. Majumder PK, Yeh JJ, George DJ, et al: Prostate intraepithelial neoplasia induced by prostate restricted Akt activation: The MPAKT model. Proc Natl Acad Sci USA 100: 7841-7846, 2003.

14. Pina F, Botelho F, Lopes T, et al: Can serum angiogenin be used to improve the diagnostic performance in prostate cancer screening? Eur J Cancer Prev 23: 166-172, 2014.

15. Katona TM, Neubauer BL, Iversen PW, Zhang S, Baldridge LA and Cheng L: Elevated expression of angiogenin in prostate cancer and its precursors. Clin Cancer Res 11: 8358-8363, 2005.

16. Yoshioka N, Wang L, Kishimoto K, Tsuji T and Hu GF: A therapeutic target for prostate cancer based on angiogenin-stimulated angiogenesis and cancer cell proliferation. Proc Natl Acad Sci USA 103: 14519-14524, 2006.

17. Olson KA, Fett JW, French TC, Key ME and Vallee BL: Angiogenin antagonists prevent tumor growth in vivo. Proc Natl Acad Sci USA 92: 442-446, 1995.

18. Olson KA, French TC, Vallee BL and Fett JW: A monoclonal antibody to human angiogenin suppresses tumor growth in athymic mice. Cancer Res 54: 4576-4579, 1994.

19. Hu GF: Neomycin inhibits angiogenin-induced angiogenesis. Proc Natl Acad Sci USA 95: 9791-9795, 1998.

20. Zhao J, Wang YC, Yang LY, Yu DH, Pan PT and Wang L: Neamine inhibits cell proliferation, migration, and invasion in H7402 human hepatoma cells. Saudi Med J 31: 1309-1314, 2010.

21. Kishimoto K, Yoshida S, Ibaragi S, Yoshioka N, Hu GF and Sasaki A: Neamine inhibits oral cancer progression by suppressing angiogenin-mediated angiogenesis and cancer cell proliferation. Anticancer Res 34: 2113-2121, 2014.

22. Yuan Y, Wang F, Liu XH, Gong DJ, Cheng HZ and Huang SD: Angiogenin is involved in lung adenocarcinoma cell proliferation and angiogenesis. Lung Cancer 66: 28-36, 2009.

23. Hirukawa S, Olson KA, Tsuji T and Hu GF: Neamine inhibits xenografic human tumor growth and angiogenesis in athymic mice. Clin Cancer Res 11: 8745-8752, 2005.

24. Majumdar MK and Majumdar SK: Isolation and characterization of three phosphoamido-neomycins and their conversion into neomycin by Streptomyces fradiae. Biochem J 120: 271-278, 1970.

25. Deng SR, Li J, Zhang ZQ, et al: DS147 improves pregnancy in mice with embryo implantation dysfunction induced by controlled ovarian stimulation. J Huazhong Univ Sci Technolog Med Sci 33: 573-580, 2013.

26. Hirukawa S, Olson KA, Tsuji T and Hu GF: Neamine inhibits xenografic human tumor growth and angiogenesis in athymic mice. Clin Cancer Res 11: 8745-8752, 2005.

27. Williams PD, Bennett DB, Gleason CR and Hottendorf GH: Correlation between renal membrane binding and nephrotoxicity of aminoglycosides. Antimicrob Agents Chemother 31:570-574, 1987.

28. Au S, Weiner $\mathrm{N}$ and Schacht J: Membrane perturbation by aminoglycosides as a simple screen of their toxicity. Antimicrob Agents Chemother 30: 395-397, 1986.

29. Yang B and Wang JJ: Industry News: Avastin approved for metastatic colorectal cancer. Discov Med 4: 79-80, 2004. 